\title{
Obstetric Outcomes and Complications After Vaginal Radical Trachelectomy: A Case Report
}

\author{
Wei Keat Tan, b, Eng Loy Tan , Lay Kok Tan ${ }^{\mathrm{a}}$
}

\begin{abstract}
We report a healthy 35-year-old Caucasian primigravida with a history of vaginal trachelectomy, cervical cerclage and laparoscopic pelvic lymphadenectomy for stage $1 \mathrm{~b} 1$ cervical cancer. She had had a spontaneous conception and received appropriate antenatal care. She presented at $23+5$ weeks gestation for leaking of liquor. Speculum examination confirmed preterm premature ruptured of membrane (PPROM) with her ultrasound showing oligohydramnios. She was admitted and despite intravenous (IV) antibiotics, developed chorioamnionitis. Emergency cesarean section was immediately performed due to maternal sepsis from chorioamnionitis. There was significant hemorrhage requiring the use of carbetocin for uterine contraction. A baby girl weighing $890 \mathrm{~g}$ was delivered via breech extraction with an Apgar score of 4 at 1 minute and 6 at 5 minutes of birth. The baby was admitted into NICU after intubation due to poor respiratory effort and developed complications of extreme prematurity, such as respiratory disorders, cardiovascular malformations, neurological and ophthalmological complications. Maternal post-operative recovery had also been complicated by wound infection that required extended antibiotics and regular wound care.
\end{abstract}

Keywords: Trachelectomy; Obstetric complications; Pregnancy

\section{Introduction}

Cervical cancer is the third most commonly diagnosed gynecological cancer worldwide, $40 \%$ of which are diagnosed in women of childbearing age $[1,2]$. Traditionally, the standard of care for a patient diagnosed with cervical cancer is radical hysterectomy with pelvic lymphadenectomy or chemoradiotherapy, both of which will lead to loss of fertility potential. As

Manuscript accepted for publication November 23, 2015

aDepartment of Obstetrics and Gynaecology, Singapore General Hospital, Singapore

${ }^{\mathrm{b}}$ Corresponding Author: Wei Keat Tan, Department of Obstetrics and Gynaecology, Singapore General Hospital, Outram Road, 169608, Singapore.

Email: andy_twk@yahoo.com

doi: http://dx.doi.org/10.14740/jmc2382w more women are delaying their childbearing for professional and financial reasons, many wish to consider fertility sparing treatment when diagnosed with early cervical cancer. Radical trachelectomy (RT) has been commonly performed for reasons to preserve fertility in young women with early cervical cancer $[3,4]$. Case series suggest that RT is as efficacious as for the standard surgery of radical hysterectomy for the treatment of early cervical cancer with the added advantage of preserving fertility [5-13]. The pregnancy rates following RT are encouraging; however, the resulting obstetric complications such as miscarriage, preterm labor, preterm premature ruptured of membrane (PPROM) and severe neonatal prematurity [2] can be devastating. Before undergoing fertility sparing surgery, all women and their partners should be fully informed of their options for treatment, the risks of surgery, success and failure rates $[14,15]$. The risk of premature labor is up to $20 \%$ of cases owing to ascending chorioamnionitis. This is attributed to the lack of cervical mucus plug after the cervix has been removed. Anatomically and physiologically, the cervix is important and necessary to conceive, hold a pregnancy and retain the pregnancy to term [15]. Therefore, all pregnancies after RT are high risk for early pregnancy loss and preterm delivery. We present a case of a pregnancy conceived after vaginal RT complicated with PPROM and chorioamnionitis.

\section{Case Report}

Madam L is an otherwise healthy 35 -year-old Caucasian primigravida with a history of having had a vaginal trachelectomy, cervical cerclage and laparoscopic pelvic lymphadenectomy done in April 2012 for stage 1b1 cervical squamous cell carcinoma. She had had a spontaneous conception and received antenatal care privately. She presented at $23+5$ weeks gestation for leaking of liquor. Speculum examination confirmed PPROM with an ultrasound showing oligohydramnios. She was admitted and given intravenous (IV) antibiotics (augmentin, ceftriaxone, metronidazole and erythromycin) for duration of 10 days before subsequently changing to IV vancomycin, amikacin and clindamycin at the subsequent development of signs and symptoms of chorioamnionitis.

She was then transferred to a public sector tertiary care center at $25+4$ weeks gestation for further management in view of extreme prematurity. An obstetric ultrasound growth scan 
was done upon her arrival and showed an appropriately grown fetus with breech presentation. She was febrile and tachycardic with uterine tenderness. Emergency cesarean section was immediately performed due to maternal sepsis from chorioamnionitis. Risks of extreme prematurity were discussed with the multi-disciplinary team of neonatologists and perinatalogists. A pfannenstiel incision was made, and the cesarean section was carried out under regional anesthesia. Intra-operative findings reveal a broad lower segment. Decision was made to proceed with a transverse lower segment cesarean section. A baby girl weighing $890 \mathrm{~g}$ was delivered via breech extraction with an Apgar score of 4 at $1 \mathrm{~min}$ and 6 at $5 \mathrm{~min}$ of birth. There was significant hemorrhage requiring the use of carbetocin for uterine contraction. Uterine incision was repaired in two layers. The baby was subsequently transferred to NICU after intubation due to poor respiratory effort.

Placental swabs for culture and histology were sent in light of signs of severe chorioamnionitis. IV prophylactic antibiotics were continued post-operatively in order to prevent endometritis and risk of post-partum hemorrhage. The histology confirmed chorioamnionitis and funicitis with neutrophilic infiltration. Placenta culture grew Pseudomonas aeruginosa and Enterococcus faecalis sensitive to aztreonam and vancomycin respectively. At post-operative day 4, IV aztreonam and vancomycin were initiated due to rising serum C-reactive protein and total white cell count. Her post-operative recovery had been complicated by wound infection that required extended antibiotics and regular wound care in a hyperbaric clinic.

Madam L's daughter also developed complications of extreme prematurity, with respiratory disorders such as hyaline membrane disease, pulmonary hypertension and bronchopulmonary dysplasia; cardiovascular malformation such as patent ductus arteriosus; neurological problems such as meningitis and cerebral ischemic infarct; ophthalmological complications such as retinopathy and corneal perforation; and systemic Pseudomonas sepsis. Her prognosis was guarded and best supportive care was discussed.

\section{Discussion}

Cervical cancer is commonly diagnosed in women of childbearing age years [2]. With earlier cervical cancer detection in women, fertility sparing surgery is increasingly performed. A series of studies [16] revealed the recurrence rate for cervical cancer to be $5.8 \%$ for radical vaginal trachelectomy versus $4.4 \%$ for radical hysterectomy. However, there was no significant statistical difference. The 5-year survival in both groups was $97 \%$ [17]. Since the recurrence rate is low, women who wish to maintain their fertility can opt for such treatment.

Literature review has shown that pregnancies are possible after RT, with $41-79 \%$ of women able to conceive subsequently [17]. However, the obstetric outcomes reported an increased incidence of preterm labor, chorioamnionitis, PPROM and miscarriages. Only $70-75 \%$ of pregnancies delivered at term. The rate of miscarriage in first trimester was quoted at $16-20 \%$, similar to background population, but second trimester miscarriage rate was higher at $8-10 \%[17]$.
Undoubtedly, RT attributes to the increase in incidence of obstetric complications. However, no specific consensus or pregnancy management guideline after cervical surgery exists. Therefore, all pregnant women with history of cervical surgery should be followed up vigilantly by a high-risk obstetric team to reduce the risks and provide early intervention should complications arise, to ameliorate the situation.

There is no strong evidence in current literature that fetal growth and placental function is affected with respect to the neovasculization [18] and healing process after an RT. In general, in an RT, cervix and upper vagina with a surrounding tissue of $1-2 \mathrm{~cm}$ cuff of paracervical tissues are removed. The descending branch of uterine artery is divided below the ureter. Once the specimen is fully mobilized, the cervix is amputated from the body of the uterus. An isthmic cerclage is then applied using non-absorbable suture. The main branch of uterine vessels is left undisturbed, it is therefore unlikely that the uterus and endometrium blood supply will be compromised. Women are advised not to conceive at least for the next 6 months [15]. Due to the absence of cervix, premature labor, PPROM and chorioamnionitis are not uncommon. Various measures have been proposed such as Wurm suture and Saling procedure to prevent ascending infection, this is however not promising due to subsequent anastomosis results in scaring and fibrosis [15].

PPROM is a recognized complication and should be treated with 10-day course of erythromycin alone as a prophylactic antibiotic [18]. This has shown a statistically significant reduction in chorioamnionitis as well as a decrease in the number of babies born within $48 \mathrm{~h}$ and 7 days of PPROM onset [19]. At present, there is no evidence to suggest the use of broad-spectrum antibiotics such as co-amoxiclav or in combination, as they have been associated with an increased risk of necrotizing enterocolitis [19, 20]. In addition, prolonged and over-zealous antibiotic regimes may inadvertently lead to emergence of nosocomial infection in pregnant women with PPROM and in neonates. PPROM may be preceded by infection which most commonly ascends from the lower genital tract [21]. Hence, screening for urinary tract infection, genital infections or even sexually transmitted infections should always be considered.

In 2003, Kenyon et al presented a systematic review which consists of 19 randomized controlled trials involving over 6,000 women and babies. The aim was to evaluate the immediate and long-term effects on maternal and fetal morbidity as well as childhood development, when antibiotics are administered to women with PPROM before 37 weeks. The results showed that there was a significant reduction in chorioamnionitis and a delay in delivery, and therefore support the use of antibiotics in PPROM. However, co-amoxiclav should be avoided because of increased risk in necrotizing enterocolitis. Royal college of Obstetricians and Gynaecologists Greentop Guideline number 44, published in November 2006, also suggests oral erythromycin as the prophylactic antibiotic in PPROM.

Another case study conducted by Casetta et al in year 2003 looked at the emergence of nosocomial Pseudomonas aeruginosa colonization/infection in pregnant women with PPROM and in neonates. Of the 63 women, 11 patients $(17.5 \%)$ acquired $P$. aeruginosa vaginal colonization during hospitalization. In a multivariate analysis, the duration of antibiotic treatment was one of the independent risk factors for acquisition 
of $P$. aeruginosa carriage. Of most concern, neonates of colonized mothers are associated with increased risk of fulminant sepsis and mortality. Most importantly attempt must be made to avoid broad spectrum antibiotics in PPROM as studies have shown that this encourages Pseudomonas infection. Instead of attempting to prolong the pregnancy in a septic environment for the fetus, delivery should be considered even at borderline viability when promising neonatal support is available. Advice from the high-risk obstetric team should be sought and timing of delivery was planned in pregnancies complicated with PPROM, should chorioamnionitis arise despite appropriate treatment of antibiotics. In Madam L's case, once PPROM and chorioamnionitis was confirmed, delivery plan was made.

In the absence of clinical signs of chorioamnionitis, delivery should be considered at 34 weeks of gestation. Immediate delivery is warranted if signs and symptoms of chorioamnionitis are present [4]. For Madam L, cesarean section was the only option for delivery due to previous RT and cerclage application. If spontaneous labor ensues, there is risk of severe antepartum hemorrhage from cervical tear with lateral extension to uterine vessels that may lead to maternal compromise. Shepherd emphasized in his paper [15] that in any woman with a history of RT, delivery must be done via a classical cesarean section using a low vertical incision owing to the absence or extreme narrowing of the lower segment. In addition, this area is usually significantly fibrosed. If a transverse incision is made into the "presumed" lower segment, one runs the risk of lateral extension and tearing through the uterine vessels causing major hemorrhage [15].

In our case, a transverse cesarean section was chosen as the lower segment was judged to be sufficiently broad. This was successfully performed without any major complications. The method of cesarean section should be individualized on case by case basis. As obstetricians will encounter more of such cases, a comprehensive protocol should be formulated.

\section{Conclusion}

Due to the increasing feasibility of the RT and favorable pregnancy rates, we will encounter more cases in the future. At present, there is no general consensus on how pregnancies should be managed under such circumstances. However, expert opinion suggests all pregnancies after RT should be managed in high risk obstetric clinic with close surveillance owing to its recognized obstetric complications. In some studies, attempts have been made to leave part of the endocervix intact while others have tried closure of the cervical os to prevent ascending infection and pre-term labor. These have not been proven successful and some centers may provide such options if there are such expertise available. Despite all efforts and attempts to prevent premature labor and PPROM, such obstetric complications may still arise. These should be managed based on individual institution guidelines and plan for delivery should be discussed despite extreme prematurity. Erythromycin should be given in cases of PPROM based on the RCOG green-top guidelines and cesarean section arranged in the event of chorioamnionitis. While classical cesarean section with a low vertical incision is recommended due to the risk of lateral extension of a transverse incision leading to uterine vessel injury and massive hemorrhage. The decision should be individualized on case by case basis and senior obstetrician input is necessary.

\section{Grant}

None.

\section{Conflict of Interest}

None.

\section{References}

1. Gizzo S, Ancona E, Saccardi C, Patrelli TS, Berretta R, Anis O, Noventa M, et al. Radical trachelectomy: the first step of fertility preservation in young women with cervical cancer (Review). Oncol Rep. 2013;30(6):2545-2554.

2. www.Uptodate.com, Fertility-sparing surgery for cervical cancer, by Marie Plante MD, Editors :Barbara Goff, MDSusan M Ramin, MD, Sandy J Falk, MD, FACOG.

3. AP Manjunath, TEJ Ind, JH Shepherd, Place of fertility sparing surgery in young women with cervical cancer, Journal of Obstetrics and Gynaecology of India Wol.57, No2; March/April 2007, pg 113-123.

4. Sean Kehoe, Eric Jauniaux, Pierre Martin-Hirsch, Cancer and reproductive Health, Chapter 7: Fertility-preserving surgery in women with cancer of the cervix, pg 87-98.

5. Shepherd JH, Crawford RA, Oram DH. Radical trachelectomy: a way to preserve fertility in the treatment of early cervical cancer. Br J Obstet Gynaecol. 1998;105(8):912916.

6. Shepherd JH, Mould T, Oram DH. Radical trachelectomy in early stage carcinoma of the cervix: outcome as judged by recurrence and fertility rates. BJOG. 2001;108(8):882885.

7. Roy M, Plante M. [Radical vaginal trachelectomy for invasive cervical cancer]. J Gynecol Obstet Biol Reprod (Paris). 2000;29(3):279-281.

8. Roy M, Plante M, Renaud MC, Tetu B. Vaginal radical hysterectomy versus abdominal radical hysterectomy in the treatment of early-stage cervical cancer. Gynecol Oncol. 1996;62(3):336-339.

9. Burnett AF, Roman LD, O'Meara AT, Morrow CP. Radical vaginal trachelectomy and pelvic lymphadenectomy for preservation of fertility in early cervical carcinoma. Gynecol Oncol. 2003;88(3):419-423.

10. Schlaerth JB, Spirtos NM, Schlaerth AC. Radical trachelectomy and pelvic lymphadenectomy with uterine preservation in the treatment of cervical cancer. Am J Obstet Gynecol. 2003;188(1):29-34.

11. Dargent D, Brun JL, Roy M. La trachelectomie elargie (T.E.). Une alternative a l'hysterectomie radicale dans le traitment des cancers infiltrants developpes sur la face exteme du col uterin. J Obstet Gynecol. 1994;2:292-295. 
12. Dargent D, Martin X, Sacchetoni A, Mathevet P. Laparoscopic vaginal radical trachelectomy: a treatment to preserve the fertility of cervical carcinoma patients. Cancer. 2000;88(8):1877-1882.

13. Covens A, Shaw P, Murphy J, DePetrillo D, Lickrish G, Laframboise $\mathrm{S}$, Rosen B. Is radical trachelectomy a safe alternative to radical hysterectomy for patients with stage IA-B carcinoma of the cervix? Cancer. 1999;86(11):22732279.

14. Shepherd JH, Spencer C, Herod J, Ind TE. Radical vaginal trachelectomy as a fertility-sparing procedure in women with early-stage cervical cancer-cumulative pregnancy rate in a series of 123 women. BJOG. 2006;113(6):719724.

15. John H. Shepherd, Cervical Cancer. Best practise \& research clinical obstetrics and gynaecology. 2012; 26:293309.

16. Xu L, Sun FQ, Wang ZH. Radical trachelectomy versus radical hysterectomy for the treatment of early cervical cancer: a systematic review. Acta Obstet Gynecol Scand.
2011;90(11):1200-1209.

17. Royal College of Obstetricians and Gynaecologists, scientific impact paper No.35, Fertility sparing treatments in gynaecological cancers, 2013.

18. Umemura $\mathrm{K}$, Ishioka S, Endo $\mathrm{T}$, Baba $\mathrm{T}$, Ezaka $\mathrm{Y}, \mathrm{Na}$ gasawa K, Takahashi M, et al. Changes of uterine blood flow after vaginal radical trachelectomy (VRT) in patients with early-stage uterine invasive cervical cancer. Int J Med Sci. 2010;7(5):260-266.

19. Royal College of Obstetricians and Gynaecologist, greentop guideline No.44, Pre-term premature ruptured of membranes, 2006.

20. Kenyon S, Boulvain M, Neilson J. Antibiotics for preterm rupture of membranes. Cochrane Database Syst Rev. 2003;(2):CD001058.

21. Casetta A, Audibert F, Brivet F, Boutros N, Boithias C, Lebrun L. Emergence of nosocomial Pseudomonas aeruginosa colonization/infection in pregnant women with preterm premature rupture of membranes and in their neonates. J Hosp Infect. 2003;54(2):158-160. 